\title{
Author Index for Volume 97
}

Aaseb $\varnothing$, U 283

Abnet, CC 123, 1567

Abrams, JA 1606

Adam, P 949

Adami, H-O 1570

Adamkova, L 231

Adelman, AS 140

Adigbli, DK 502

Adjadj, DJ 883

Agalliu, I 826

Agapitos, E 1124

Agelaki, S 43

Aguayo, F 85

Ahn, IS 1655

Ahn, JB 458

Ahn, JS 22

Ahn, MJ 22

Ahn, Y-C 22

Aikou, T 405

Aitini, E 1613

Akaike, H 494

Akiba, S 85

Akiyama, F 654

Alami, N 58

Al-Batran, S-E 177

Alderden, RA 194

Alexandre, J 290

Alfaro, J 1206

Ali, S 761

Allen, J 1225

Allgayer, H 1432

Alonso-González, C 755

Alston, RD 1588

Alves, F 1432

Amado, RG 1469

Amant, F 1194

Amaratunga, G 327

Andera, L 73, 1716

Andersen, TT 327

Anderson, JH 1266

Andersson, S-O 730

Anderton, JA 267

Andrén, O 730

Andres-Beck, B 1322

Andreuccetti, M 1613

Andries, L 1344

Androulakis, N 43

Angibaud, P 1344

Angus, B 29

Ansell, P 1310

Ansell, W 308, 1188

Antoni, G 1046

Antonuzzo, A 1613

Aozasa, K 952

Aramin, N 1432

Araya, T 778

Arcaro, KF 809

Arena, MG 593

Arguin, G 1505

Aridome, K 405

Armesilla, AL 745

Artru, P 297
Arts, J 1344

Arvanitakis, M 582

Asakawa, T 162

Asterling, S 308, 1188

Astone, A 1035

Astudillo, A 201

Atmaca, A 177

Attard, G 1338

Atz, J 315

Atzpodien, J 1329

Aubard, Y 883

Aubert, C 919

Aubert, J-P 345

Audoynaud, C 1218

Auperin, A 1664

Autschbach, F 1271

Auvinen, A 115

Auvrignon, A 238

Averill, J 646

Avery-Kiejda, KA 1225

Aviv, A 1696

Axelsson, O 1583

Baas, P 276

Baas-Thijssen, MCM 717

Bagnardi, V 802

Bahra, M 523

Bailey, A 283

Bain, C 1295

Bajetta, E 1469

Ball, J 1696

Ballardini, M 1499

Bamias, A 1124

Bannon, JH 1234

Baptiste, C 1505

Barbachano, Y 670

Barbieri, R 1613

Bardadin, K 531

Bardin-Mikolajczak, A 832

Barduagni, M 1040

Barker, SE 210

Baron, A 391

Barone, C 1035

Bart, J 322

Bartelink, H 712

Bartlett, JMS 378

Barwell, J 1696

Basso, M 1035

Bast, A 1084

Bates, DO 223

Batra, SK 345

Baumann, H 1513

Bearzi, I 92

Bedke, J 1271

Beebe, K 741

Beer, A 1146

Belanger, S 58

Belderbos, J 276

Belli, F 1040

Benedict, A 152

Benhadji, K 628

Bennett, JA 327
Bennouna, J 297

Benyoub, A 883

Beral, V 434

Berardi, R 92

Bercovich, E 1499

Berg, J 1696

Berger, MR 1106

Bergkvist, L 105

Bergström, A 429

Berkhof, J 1084

Berney, D 1188

Berteloot, P 1194

Bertheau, P 1157

Bertholon, J 1218

Betts, G 1017

Birch, JM 1588

Birnboim, HC 1242

Bishop, DT 1701

Bishop, H 725

Bismar, TA 730

Bissett, D 844

Blagden, SP 1338

Blanc, J-F 862

Blanquicett, C 919

Bleijenberg, G 612

Bobrow, M 486

Bodempudi, V 513

Bodoky, G 1469

Body, JJ 964

Boehm, M 523

Bogdanova, T 818

Bogdanovic, G 992

Bohic, S 194

Boige, V 862

Bole, C 14

Bollet, MA 1046

Bonezzi, K 888

Bonnier, P 883

Boot, H 712

Borbath, I 582

Borrow, R 989

Bosetti, C 995

Bottiglieri, L 802

Bottomley, A 302

Bouché, O 862

Bouchet, BP 1218

Boudny, V 231

Bourdon, J-C 277

Bourhis, J 1664

Bozec, A 65

Bozionelou, V 43

Braly, PS 1618

Bray, D 670

Bremnes, RM 283

Brenner, H 1486, 1597

Breuzard, G 562

Brewster, DH 999

Briasoulis, E 1560

Bridle, H 247

Brinton, LA 832

Brisse, H 238

Broderick, P 1305
Bronzwaer, JGF 1084

Brown, EJ 1189

Brown, TJ 358

Brücher, BLDM 1404

Bruynzeel, AME 1084

Buffler, PA 1315

Bugat, R 297, 857

Bukawa, H 792

Bulliard, J-L 1013

Burkholder, I 1480

Burton, C 1310

Buse, S 1271

Butler, P 1601

Butow, PN 6

Büchler, MW 598

Buxton, ILO 1372

Byrne, P 1333

Cafaro, V 1354

Cairncross, JG 302

Cal, S 201

Calgiuri, M 1598

Callaghan, R 194

Calvo, F 628

Calvo, JC 1683

Cameron, DA 479

Campbell, C 1601

Campbell, RB 910

Camplejohn, R 1696

Campo, L 1277

Camponovo, A 1139

Campos, S 1618

Capetillo, M 85

Carén, H 1416

Carles, J 1206

Carlsen, K 1020

Carmi, VD 1655

Carmon, L 1655

Carpenter, B 576

Carter, N 1696

Casas, G 1683

Cascinu, S 92

Casey, G 557

Cassano, A 1035

Cassidy, J 745

Castellone, MD 1545

Castera, D 1200

Castillo, A 85

Catalan, G 1206

Cats, A 712

Cavalli, F 1139

Cave, J 472

Celetti, A 1545

Celsing, F 769

Cen, L 785

Chakrabarti, M 1266

Chan, AT 1295

Chan, FKL 895

Chan, JK 605

Chan, K-K 1409

Chan, K-W 1409

Chan, S 1618 
Chan, Y-P 1409

Chandler, I 1305

Chang, JS 1315

Chang, J-Y 334

Chang-Claude, J 1486

Chanock, SJ 832

Chanudet, E 949

Chapelin, D 1157

Chatelut, E 290

Chaturvedi, P 345

Chaudhuri, K 574

Chauvenet, L 1200

Chen, C-C 334

Chen, C-S 785

Chen, G-s 420

Chen, J 1577

Chen, L-T 334

Chen, W 123

Cheng, I 557

Cheng, Y 1441

Cheng, YY 895

Cheung, MK 605

Chevrette, M 941

Chiao, J 628

Chien, C-R 275

Chilvers, C 434

Choi, IJ 700

Choi, KS 700

Choi, YS 22

Chou, W-C 877

Choudhury, A 105, 769

Chrousos, GP 637

Chu, Y-Y 1409

Chung, HC 458

Ciccolini, J 919

Clarke, SJ 1071

Clavère, P 883

Clézardin, P 964

Clements, K 725

Clifford, SC 267

Cnattingius, S 1583

Cochrane, BB 98

Coens, C 302

Coffey, J 1701

Cognettti, F 1015

Cohn, D 605

Colditz, GA 1295

Coleman, D 1601

Coleman, MP 999, 1009

Colleoni, M 802

Colman, L 14

Comandini, D 1469

Conte, P 1469

Cooke, TG 1632

Cooper, M 844

Cooper, R 1333

Coppes, RP 322

Corbishley, C 670

Corte, MD 957

Corvalan, A 85

Cos, S 755

Costanzo, ES 1625

Cotton, SC 133

Coudert, B 857

Coudry, RA 678

Couturier, J 238

Cowan, C 705

Craven, I 1333
Crellin, A 1333

Crnković-Mertens, I 1271

Crossley, B 434

Crouch, S 1310

Crozier, JEM 1266

Cruickshank, ME 133

Culine, S 857

Cunha, IW 678

Cvitkovic, E 628

Czarnocka, B 531

D'Alessandro, C 802

D'Alessio, G 1354

D'Argento, E 1035

d'Enghien, CD 238

D’Ottavio, AM 1040

Dacre, J 472

Dahan, L 862

Daisaki, H 1493

Dalton, P 670

Dalton, SO 1020

Darling, JL 745

Das, T 1361

David, O 1552

Davies, ML 384

Davies, T 464

Daw, NC 1552

Dawsey, SM 123, 1567

Day, JM 1673

de Bono, JS 1338

de Groot, DJA 1077

de Jong, S 1077

de Jonge, M 1338

De Knijf, A 1194

De Lorenzo, C 1354

de Roquancourt, A 1157

De Rosa, G 1545

de Thé, H 1157

de Vries, EGE 322, 1077

de Witte, O 302

Debled, M 1642

DeFreest, LA 327

Delattre, O 238

Delavault, P 253

Dellapasqua, S 802

Delord, J-P 297

Delys, L 818

Denkert, C 523

Derkow, K 769

Desseigne, F 297

Detours, V 818

Deutsch, E 1664

Devercelli, G 1469

Dewar, J 725

Dewhirst, MW 735

Di Benedetto, M 1545

Di Lauro, L 593

Diamond, A 826

Dietel, M 523

Dillner, J 129

DiMarsico, R 1613

Dimopoulos, M-A 1124

Dirix, L 1165

Dirix, LY 659

Discalzi, G 1300

Dittrich, C 1480

Divitini, M 686

Dixon, JM 1182
Do, M-S 1655

Docherty, Z 1696

Dodwell, D 725, 1211

Domenech, M 1206

Donamaria, C 1642

Dong, Z-W 123, 1567

Doriath, V 964

Douillard, JY 297

Douillard, J-Y 857

Drabik, G 589

Du, M-Q 949

Duan, D 1441

Duan, R-D 1441

Dubbelman, R 712

Ducreux, M 862

Dudakia, D 1701

Dudek, AZ 513

Dufer, J 562

Dumont, JE 818

Dunn, DBA 223

Dupouy, N 862

Durand, M 1642

Düring, M 1020

Easton, DF 1701

Eccles, S 670

Eden, TOB 1588

Edler, L 1480

Edwards, J 378

Efficace, F 302

Eijkemans, MJC 868

Eisen, T 247

Eisenbach, L 1655

Eizuru, Y 85

Ejeskär, K 1416

el Galta, R 247

El-Khoury, V 562

Elliott, F 1701

Ellison, DW 267

Elomaa, I 1028

Emery, J 486

Encioiu, E 1690

Enders, GA 1388

Ernst, T 1475

Errami, A 1457

Espié, M 1157

Esposito, I 1106

Etienne, LL 1139

Evans, TRJ 745

Faivre, S 628

Falcone, A 1613

Falette, N 1218

Fall, K 730

Fallowfield, L 152

Fan, ST 50

Fanciullino, R 919

Fanshawe, T 486

Farooqui, M 1523

Farooqui, N 502

Faroux, R 297

Farrar, WL 1116

Fear, NT 1315

Fegan, C 253

Feingold, D 1606

Felici, A 1015

Fellinger, K 315
Ferretti, G 1015

Feskanich, D 1295

Feugeas, J-P 1157

Fichtner, I 1234

Figueroa-Moseley, C 14

Fiori, M 1499

Fizazi, K 857

Floquet, A 1642

Floren, W 1344

Fluck, M 1329

Foggi, P 593

Fojtova, M 231

Foley, J 183

Fong, PC 1338

Fonseca, FP 678

Font, A 1206

Fontana, A 1613

Fontana, E 1613

Forman, D 1211, 1393

Formento, P 65

Forsyth, P 302

Foster, PA 1673

Fountzilas, G 637

Fourquet, A 1046

Fourré, N 562

Fr Brunsvig, P 283

Franc, B 818

François, E 297

Franceschi, S 995

Francque, S 582

Fransson, S 1416

Frapolli, R 888

Frascogna, V 1664

Frassineti, GL 1499

Frattini, M 1139

Fraumeni, JF 1577

Freshney, RI 1714

Fridkin, M 1655

Friess, H 1106

Fritschi, L 686

Frost, A 1480

Fuchs, CS 98

Fujii, H 494

Fujimura, M 778

Fujita, H 1425

Fujita, N 1399

Fujita, Y 426

Fukushima, M 37

Fukuta, N 1532

Furnival, C 1251

Fushimi, K 792

Gakiopoulou, H 1124

Gallardo, E 1206

Galli, C 1613

Galli, L 1613

Galli, V 1545

Gallimore, A 1017

Galmarini, CM 628, 1218

Gamucci, T 1040

Gao, Y-T 1577

García-Muñiz, JL 957

García-Suárez, O 201

Garcia-Closas, M 832

Gardner, J 1696

Garnero, P 964

Garpestad, OK 283 
Gatenby, RA 646

Gatter, KC 1277

Gauffin, F 992

Gautier, H 1200

Geerts, T 1344

Gekeler, V 523

Genet, D 883

Georgekutty, J 327

Georgiou, A 1696

Georgoulias, V 43, 1560

Geraci, M 1588

Ghisini, R 802

Ghisletta, M 1139

Ghoul, A 628

Giacometti, S 919

Giassas, S 43

Giavazzi, R 888

Gielissen, MFM 612

Giese, T 1106

Gilbertson, RJ 267

Gillessen, S 1480

Gillies, RJ 646

Gilmore, JL 183

Giovannini, N 1499

Giovannucci, EL 98

Gires, O 315

Giuliante, F 1035

Givalos, N 1124

Glatstein, E 452

Gnad-Vogt, U 1475

Gnant, M 1021

Górka, B 531

Godkin, A 1017

Goehrig, D 964

Goldbohm, RA 1291

Goldhirsch, A 802

Goldie, SJ 1322

Goldstein, D 464

Goldwasser, F 290

Gonterman, RM 183

González, A 755

González, LO 957

González, MV 201

González-Larriba, JL 1206

Gonzalez del Alba, A 1206

Goodchild, R 486

Gooding, LR 992

Gore, ME 1618

Goteri, G 949

Göttlicher, M 177

Gozgit, JM 809

Graf, MR 619

Grann, VR 1606

Granstam-Björneklett, H 105

Gray, R 1305

Green, SR 628

Gregory, CA 1552

Greil, R 1021

Gretz, N 1432

Greving, JP 429

Grieshaber, CK 58

Griffin, RJ 513, 1523

Groen, HJM 322

Grosse, SM 210

Gröne, EF 1271

Groves, FD 140

Grützmann, R 1432

Guarino, V 1545
Guastalla, J-P 1200

Guichard, F 1200

Gunelli, R 1499

Guo, X 745

Gupta, K 1523

Gurda, A 589

Gustafsson, B 992

Haddad-Guichard, Z 1200

Haferkamp, A 1271

Hagiwara, S 1532

Haglund, B 1583

Haim, EB 1655

Hale, GA 539

Hall, MD 194

Hamaguchi, T 170

Hamamura, RS 1099

Hamashima, C 1493

Hambley, TW 194

Han, J-H 22

Han, T-Q 1577

Hanauske, A-R 1480

Handgretinger, R 539

Hansson, BG 129

Harada, K 1425

Harada, M 1648

Haraguchi, N 543

Harper, SJ 223

Harrington, KJ 670

Harris, AL 1277

Hart, SL 210

Hartley, JA 253, 927

Harvey, J 464

Harvey, JR 761

Hasumi, K 1058

Haug, U 1597

Hautmann, R 1146

Hawinkels, LJAC 398

Hawkins, MM 695

Hayakawa, S 1532

Hebbel, RP 513

Heidrich, B 1432

Heinzel, T 177

Helander, I 105

Helbekkmo, N 283

Henderson, BW 1513

Hendrikse, NH 322

Henken, FE 1457

Hentsch, B 177

Herbst, F 1021

Hermon, C 434

Hersey, P 1225

Hershman, DL 1606

Hertervig, E 1441

Hickok, JT 14

Higashi, M 85

Higo, M 792

Hilakivi-Clarke, L 1570

Hildebrand, D 1146

Hirakawa, K 550

Hiraoka, S 1425

Hirata, K 851

Hjelde, HH 283

Ho, CSK 1251

Ho, JWY 50

Ho, YT 1673

Hochhaus, A 1475, 1480

Hodgson, S 1696
Hoel, DG 140

Hofbauer, F 1021

Hoffmeister, M 1486

Hofheinz, R-D 1475

Hofman, P 65

Hofmann, H-P 523

Hohenfellner, M 1271

Holladay, MS 539

Holmen, J 112

Homma, S 1053

Hommes, DW 398

Hong, L 420

Hoover, RN 688

Hoppe-Seyler, F 1271

Hoppe-Seyler, K 1271

Horgan, K 1211

Horgan, PG 1266

Horii, J 1425

Horii, R 654

Horwitz, EM 1552

Houbiers, G 582

Houédé, N 1642

Houlston, R 1305

Houlston, RS 247, 1449

Hövelmann, S 177

Hoye, NA 29

Hsieh, AC 453

Hsieh, F-C 785

Hsieh, H-P 334

Hsing, AW 1577

Hu, C-Y 877

Huang, W 992

Hubner, RA 1449

Huddart, RA 1701

Hulsey, TC 140

Humblet, Y 1469

Huon, I 238

Hurst, NG 971

Hurt, EM 1116

Husain, AN 368

Husseini, F 297

Hutchison, C 705

Hutson, A 1513

Iizuka, N 1399

Ikeda, M 170

Imoto, I 260

Imre, G 523

Inazawa, J 260

Innes, KE 688

Ino, K 1538

Inoue, H 543

Inoue, M 446

Iovine, R 1545

Ishigami, T 792

Ishihara, Y 1648

Ishitsuka, H 1399

Ishiwata, R 37

Ismail, T 971

Ito, M 260

Ito, $\mathrm{N} 260$

Ito, Y 654

Itoh, K 1648

Itoh, T 85

Iwasaki, LM 826

Iwasaki, M 446

Iwase, T 654

Iyengar, R 539
Jack, A 1310

Jack, W 479

Jacob, J 523

Jacobson, HI 327

Jacobson, JS 1606

Jagadeeswaran, R 368

Jagoditsch, M 1021

Jain, M 118, 1600

Jakesz, R 1021

Janicot, M 1344

Janin, A 1157

Jansen, EPM 712

Janssen, L 1344

Janssens, B 1344

Jaramillo, M 1361

Jarkovsky, J 231

Jasinski, P 513

Jäger, D 598

Jäger, E 177

Jäger, M 315

Javed, MA 1372

Jenkinson, HC 695

Jeschke, U 637

Jesnowski, R 1106

Jeung, H-C 458

Jia, W 619

Jiang, X 1106

Jin, VX 895

Jit, M 989

Joel, SP 308, 1188

Joensuu, H 1028

Johansen, C 1020

Johansson, J-E 730

Johnson, DL 1344

Jolly, KW 1315

Jondal, M 769

Jones, A 472

Jones, D 152

Jones, GL 29

Jones, RB 391

Journe, F 964

Jouve, J-L 862

Juin, P 883

Jung, KW 700

Jung, M 1344

Junquera, S 957

Juste, S 58

Kabat, GC 118, 1600

Kadowaki, T 260

Kajiyama, H 1538

Kakugawa, Y 1493

Kalantaridou, SN 637

Kalikaki, A 1560

Kalra, AV 910

Kalykaki, A 43

Kamangar, F 1567

Kameoka, S 1277

Kaminsky, M-C 857

Kamoto, T 260

Kanazumi, N 1260

Kaniwa, N 145

Kapp, DS 605

Karlins, E 826

Karlsson, M 730

Karnon, J 479

Kasahara, K 778

Kasprzyk, PG 1673 
1720

Kasuya, H 1260

Kato, BS 1696

Kato, J 851, 1425

Kato, K 170, 1260

Kato, T 1277

Kato, Y 792

Katsuragi, K 550

Kawaguchi, Y 494

Kawanishi, H 260

Kaye, S 1618

Kayed, H 1106

Ke, Y 218

Kearins, O 725

Keh, C 971

Keleg, S 1106

Kentepozidis, N 43

Kerr, GR 479

Kesterton, I 1696

Khanna, RC 14

Kieler, H 1583

Kikkawa, F 1538

Kim, J 22

Kim, JJ 1322

Kim, K 22

Kim, ST 22

Kim, YS 741

Kimura, H 778

Kimura, M 1532

Kimura, T 1277

King, P 1344

Kinmonth, AL 486

Kinnon, C 210

Kinoshita, T 1260

Kirby, JA 761

Kitazawa, S 1707

Kitzen, J 1338

Kiyosawa, K 145

Kleeff, J 1106

Klijanienko, J 238

Klotz, LH 1690

Knuiman, M 686

Knuth, A 177

Kobayashi, C 1099

Kobayashi, M 1277

Kogner, P 1416

Koizumi, F 778

Kolodziejczyk, P 589

Kolonel, LN 440

Koltz, PF 183

Komohara, Y 1648

Konger, RL 183

Kono, K 494

Kopp-Schneider, A 1146

Korbelik, M 1381

Koriyama, C 85

Korkolopoulou, P 1124

Korpela, R 1028

Koshiol, J 1567

Kotani, K 426

Kotasek, D 464

Kothonidis, K 73, 1716

Kouri, M 1028

Koutsopoulos, A 1560

Kouzu, Y 792

Kovarik, A 231

Kovarik, J 231

Kozu, T 1493

Krasner, CN 1618
Kripp, M 1475

Kristiansen, G 523

Kritz, A 210

Krumroy, LM 557

Krupa, K 1632

Kubben, FJGM 398

Kudo, M 1532

Kudoh, S 162

Kuhn, A 1146

Kuhn, E 888

Kuipers, EJ 868

Kulig, J 589

Kunikane, H 162

Kunitoh, H 162

Kuo, C-C 334

Kurahara, H 405

Kurashina, R 1099

Kurdistani, SK 1

Kuroiwa, G 851

Kurozawa, Y 426

Kut, C 978

Kutner, R 1552

Kwon, EM 826

La Vecchia, C 1300

Labourey, JL 883

Lacoste, J 941

Ladekarl, M 1135

Lagiou, P 1570

Lala, PK 1090

Lamblot, C 1218

Lamelas, ML 957

Lamers, CBHW 398

Landi, L 1613

Langlet, P 582

Langrehr, J 523

Lansdown, M 1180

Laplanche, A 857

Larsson, SC 1005

Lassalle, S 65

Lauerova, L 231

Laughner, R 183

Law, SY-K 1409

Law, WE 1372

Lawrence, G 725

Lawson, AB 140

Le François, BG 1242

Le, TKP 1077

Le Tourneau, C 628

Lebrun-Ly, V 883

Lecluse, Y 1664

Ledermann, JA 927

Leduc, B 1200

Lee, AK 700

Lee, C-Y 877

Lee, H-S 22

Lee, J 22

Lee, JE 429

Lee, K-S 22

Lee, M-J 741

Lee, N 1552

Lee, SY 700

Lee, TK 50

Leek, RD 1277

Leese, MP 1673

Legrès, L 1157

Leitch, EF 1266

Lejeune, C 883
Lekander, M 105

Lemanski, N 327

Lemay, R 1505

Lennard, TWJ 761

Lennette, ET 1567

Lepillé, D 1200

Lesimple, T 857

Leunen, K 1194

Leung, W 539

Leung, WK 895

Levi, F 995, 1013

Levidou, G 1124

Lewis, DA 183

Leyland-Jones, B 58

Li, H 420

Li, J 895

Li, KM 1071

Li, L 1432

Li, Q 420

Li, Y 1523

Lianes, P 1206

Libert, F 818

Lightfoot, T 986

Lin, H-J 785

Lin, J 785

Lin, L-I 877

Lindblad, P 429

Lindblom, A 1175

Lindemalm, C 105

Lindgren, S 1441

Lindhofer, H 315

Lindsey, JC 267

Lippens, C 65

Lissowska, J 832

Little, J 133

Liu, CL 50

Liu, J-F 334, 1449

Liu, S 308, 1188

Liu, X 557

Lo, CM 50

López, JA 1251

Loblaw, DA 1690

Locker, G 152

Logan, RFA 1449

Loizidou, M 502

Looijenga, LH 1707

Lopez, M 593

Lorch, G 183

Loria, RM 619

Lortholary, A 857

Lotze, MT 1598

Lou, L 934, 1715

Löf, M 1570

Löhr, M 1106

Lowe, H 253

Lu, YJ 1707

Luangdilok, S 670

Lubbe, S 1305

Lucenteforte, E 995

Lucraft, H 29

Lugassy, G 1655

Luini, A 802

Lukan, N 1475

Lukkien, C 429

Lusher, ME 267

Lutgendorf, SK 1625

Lynch, J 1020

Lynge, E 1019
Ma, J 98

Ma, PC 368

Mac Gabhann, F 978

Macher-Göppinger, S 1271

Machlenkin, A 1655

MacRobert, AJ 502

Madranges, N 1642

Mady, S 670

Maeda, S 405

Maemura, K 405

Maenhaut, C 818

Magni, E 802

Magnolfi, E 1040

Magnusson, C 1287

Maini, PK 646

Makrigiannakis, A 637

Man, EPS 895

Man, K 50

Mandolesi, A 92

Mansel, R 152

Manson, JE 98

Maraqa, L 1180

Marchetti, S 577

Marconi, SA 809

Margolin, S 1175

Mariën, A 1344

Marijnen, CAM 717

Mark, SD 123, 1567

Marlow, LAV 691

Marosi, C 302

Maroun, JA 1242

Marsden, HB 695

Marselos, M 43

Martínez-Campa, C 755

Martin, C 1200

Martin, J 883

Martin, JE 412

Martin, V 1139

Martinelli, M 888

Martinsson, T 1416

Martinussen, N 986

Mascolo, M 1545

Maspoli, M 1013

Massard, C 857

Masson, LF 133

Mataki, Y 405

Matakidou, A 247

Matsui, Y 260

Matsumoto, K 1053

Matsumoto, S 37

Matsumoto, T 543

Matsumura, Y 170

Matsunaga, T 851

Mattes, ML 1625

Mauer, M 302

Maughan, NJ 1393

Maurer, A 177

Mauriac, L 1642

Märten, A 598

Mavroudis, D 43, 1560

Maxwell, T 1251

Mazza, O 1683

Mazzucchelli, L 1139

Mc Gee, MM 1234

McGlynn, LM 378

McGuckin, MA 1251

McIntyre, A 1707

McKee, RF 1266 
McMahon, T 705

McMeekin, DS 1618

McMillan, DC 1266

McPherson, K 434

Mediavilla, MD 755

Meijer, CJLM 1457

Meiss, R 1683

Melcher, A 1333

Melia, J 1601

Meliffi, L 1040

Melillo, RM 1545

Mellado, B 1206

Mellor, HR 194

Mellor, P 761

Mellstedt, H 105

Mercier, C 919

Merino, AM 957

Merolla, F 1545

Merrouche, Y 857

Merx, K 1475

Metayer, C 1315

Meza, JL 345

Mhaidat, NM 1225

Mi, Y 934, 1715

Michael, M 464

Michaud, DS 98

Michon, J 238

Mikaelsson, E 105

Mikeljevic, JS 1211

Mikula, M 531

Milano, G 65

Milbauer, L 513

Miller, AB 118, 1600

Miller, E 989

Mimori, K 543

Mimura, K 494

Minami, R 1053

Minas, V 637

Mirimanoff, RO 302

Misset, J-L 1157

Missiaglia, E 1707

Mitrou, S 637

Mitsui, F 494

Miura, T 1399

Miyamoto, K 778

Miyanishi, K 851

Miyoshi, E 1538

Moasser, MM 453

Modok, S 194

Moerman, P 1194

Molinari, F 1139

Moniaux, N 345

Monroe, KR 440

Montella, M 995

Montgomery, DA 1182, 1632

Moran, A 1588

Morant, R 1480

Mori, M 543

Moribe, T 1399

Moriyama, N 1493

Morizane, C 170

Morris, E 1393

Morris, H 486

Morrow, GR 14

Mortensen, PB 1020

Moscetti, L 1040

Moss, S 1601

Mosseri, V 238
Motta, S 1545

Mould, TA 927

Mozaffari, F 105

Mross, K 1480

Mucci, LA 730

Muir, KR 1449

Mukherjee, R 378

Munakata, H 1532

Munzer, C 238

Muramatsu, Y 1493

Muro, K 170

Murphy, KJ 761

Murphy, SP 440

Murray, GI 576

Murray, S 1560

Murthy, BL 1211

Mutsvangwa, K 308, 1188

Mühlradt, PF 598

Müller-Hermelink, HK 949

Nadella, KS 183

Nagai, Y 37

Nagasaka, T 1538

Nagashima, H 851

Nagashima, M 1532

Nagengast, WB 322

Nagle, RB 646

Naito, M 426, 1648

Nakahama, H 170

Nakai, Y 952

Nakamura, Y 543

Nakao, A 1260

Nakao, S 778

Nakashima, D 792

Nakatani, T 1532

Nakayama, M 952

Nallasura, V 368

Nam, RK 1690

Naman, H 297

Nanni, O 1499

Narendran, A 327

Narod, SA 1187, 1690

Nasioulas, G 73, 1716

Natsugoe, S 405

Naucler, P 129

Navone, N 1683

Nawa, A 1538

Nawaz, F 391

Nawaz, S 391

Neal, K 133

Neckers, L 741

Nedergaard, BS 1135

Neff, JK 1432

Negri, E 995, 1300

Neill, M 1690

Neugut, AI 1606

Neuhaus, P 523

Neumann, A 177

Neven, P 1194

Newman, SP 1673

Newton, C 927

Newton, R 1310

$\mathrm{Ng}$, I 50

$\mathrm{Ng}$, KT 50

Nicholson, AG 949

Nie, L 420

Niedergethmann, M 1432
Nielsen, K 1135

Niesporek, S 523

Niessen, HWM 1084

Nieto, Y 391

Niitsu, Y 851

Nikolaidou, M 43

Nilsen, TIL 112

Nilsson, ̊ 1441

Nilsson, B 105

Ning, T 218

Nishikawa, T 1277

Nishimura, K 952

Nishio, K 426, 778

Nishiyama, H 260

Niwa, S 1577

Noguchi, M 1648

Nogué, M 1206

Noh, SH 458

Noma, H 405

Nomoto, S 1260

Nomura, H 792

Nomura, S 1538

Nonomura, N 952

Norberg, M 769

Nordgren, A 992

Nowicki, M 412

Nunziata, C 593

Nutting, CM 670

Nuzzo, G 1035

Nyengaard, JR 1135

Nygren, AOH 1457

O’Boyle, G 761

Ochi, H 1053

Odutoye, T 670

Ogawa, O 260

Ogawara, K 792

Ogimoto, I 426

Ohira, M 550

Ohnuma, H 851

Ohyashiki, JH 1099

Ohyashiki, K 1099

Oikonomou, E 73, 1716

Ojutkangas, M-L 105

Oka, D 952

Oka, M 1399

Okada, S 1053

Okamoto, H 162

Okamoto, T 851

Oki, A 1053

Okusaka, T 170

Okuyama, A 952

Olaitan, A 927

Oliver, RTD 308, 1188

Ollus, A 1028

Olson, S 1552

Olsson, A 769

O’Neill, A 1234

Ono, K 792

Oosterhuis, JW 1707

Orfeuvre, H 1200

Orlando, A 223

Ornelles, DA 992

O'Rourke, K 140

Osaka, H 550

Osann, K 605

Osoba, D 302
Osorio, LM 769

Ostrander, EA 826

Ota, T 1058

Otani, S 1260

Otani, T 446

Otis, CN 809

Ott, N 1404

Ökvist, A 769

Österborg, A 105, 769

Österlund, P 1028

Overmeer, RM 1457

Paliczka, E 531

Pallis, AG 1560

Pampillón, C 1234

Pan, W-Y 334

Pan, Y 218

Panchapakesa, V 1361

Pandeya, N 1295

Pangon, L 1696

Papaemmanuil, E 1305

Papo, NL 479

Pappagallo, GL 1499

Pappas, P 43

Paquette, B 1505

Paraiso, D 1200

Paraskevaidis, EA 637

Paret, C 1146

Park, EC 700

Park, K 22, 700

Park, KGM 902

Parker, E 1701

Parker, R 1601

Pasquini, E 1499

Paterson, J 58

Patnick, J 1601

Patsouris, E 1124

Paul, J 705

Pavlidis, N 637

Paximadas, D 1696

Paz, A 1655

Pebody, R 989

Peeters, M 582, 1469

Peinado, JR 201

Pelucchi, C 995, 1300

Penegar, S 1305

Peng, B 1116

Pentecost, BT 809

Pentland, AP 14

Peplonska, B 832

Pepper, C 253

Perrier, H 297

Peto, J 434, 1305

Petsas, G 637

Pezzella, F 1277

Pfeifer, S 1583

Piana, L 883

Piccerelle, P 919

Picelli, S 1175

Pidal, I 957

Piedbois, P 297

Pierantoni, C 92

Pierga, J-Y 1046

Pieterse, AH 717

Pignon, J-P 862

Pike, MC 434, 440

Pilarsky, C 1432 
Pileri, SA 949

Pintzas, A 73, 1716

Pinzon-Charry, A 1251

Piolatto, PG 1300

Piperi, C 1124

Pira, E 1300

Pirie, K 434

Pitkin, L 670

Pituch-Noworolska, A 589

Pivot, X 857

Plantaz, D 238

Plassa, L-F 1157

Plouffe, B 1505

Plummer, SJ 557

Pollak, MN 98

Ponce, AM 735

Poon, RTP 50

Poonawala, T 1523

Popel, AS 978

Popiela, T 589

Post, S 1432

Potter, BVL 1673

Potts, HWW 472

Powles, T 308, 1188

Pozzo, C 1035

Prevost, AT 486

Priou, F 857

Pritchard-Jones, RO 223

Pritsch, M 1271

Probert, E 1716

Prockop, DJ 1552

Proctor, SJ 29

Pronk, LC 1338

Provencher, DM 1618

Pruneri, G 802

Pugh, JL 1701

Puisieux, A 1218

Puisset, F 290

Pujade-Lauraine, É 1200

Pukkala, E 115

Purohit, A 1673

Pühringer-Oppermann, F 1404

Pye, D 761

Pyke, CM 1251

Qiao, Y-L 123, 1567

Qiu, Y 223

Qualman, SJ 785

Quirke, P 1393

Rabbani, Z 735

Rabl, H 1021

Rachet, B 999

Ranaldi, R 949

Randimbison, L 1013

Rankin, EM 844

Rantala, J 1175

Raobaikady, B 1673

Raoul, J-L 862

Raoul, V 290

Rapley, EA 1701

Rashid, A 1577

Rastogi, S 1090

Raum, E 1486

Ray-Coquard, I 1200

Raymond, E 628

Reece, W 464
Reed, MJ 1673

Regeling, A 1077

Reinier, K 1315

Reiser, J 1552

Reitz, M 1329

Renshaw, FG 1618

Reynaert, H 582

Rha, SY 458

Rhys Evans, P 670

Ribeiro, A 238

Riccardi, E 888

Richesson, D 832

Ricketts-Loriaux, RSJ 809

Rigby, H 223

Ringuette, MJ 358

Risley, P 502

Rivory, LP 1071

Roberts, GT 384

Roberts, H 1277

Roberts, JM 688

Roberts, K 1063

Robertson, R 1601

Robinson, CB 1625

Roché, H 290

Roddam, AW 434

Rodríguez, JC 957

Rodriguez-Sierra, JF 345

Rogers, T 1523

Roh, JK 458

Rohan, TE 98, 118, 1600

Rolaki, A 637

Romagnani, E 1139

Roman, E 986, 1310

Roman, SL 1625

Roose, T 194

Rose, F 646

Rose-John, S 1513

Rosenberg, LU 1287

Rosenquist, R 769

Rosero-Bixby, L 837

Rosol, TJ 183

Ross, L 1020

Roy, P 1361

Roychoudhury, P 574

Rubie, H 238

Rubin, MA 730

Rudd, MF 247

Ruf, P 315

Rumjahn, SM 1372

Ruotsalainen, T 1028

Russo, C 1315

Ruzankina, Y 1189

Ryan, JL 14

Ryd, W 129

Rylander, E 129

Sánchez-Barceló, EJ 755

Sacca, P 1683

Saetta, A 1124

Sagawa, T 851

Saijo, N 162

Saito, D 1493

Saito, H 1493

Saito, K 792

Sakaguchi, K 1425

Sakaguchi, Y 1532

Sakaida, I 1399
Sakamoto, K 1399

Sakoda, LC 1577

Sakurai, M 1053

Salahuddin, FK 735

Salek, T 1469

Saletti, P 1139

Salgia, R 368

Salmon, R 1046

Samonigg, H 1021

Sandin, S 1570

Santoro, M 1545

Sarbia, M 1404

Sasazuki, S 446

Sastre-Garau, X 1046

Sato, Y 851

Satoh, T 1053

Savage, SA 832

Savignoni, A 1046

Sawada, T 550

Saxelin, M 1028

Scartozzi, M 92

Schaberl-Moser, R 1021

Schatzlein, A 745

Scheidereit, C 523

Schellens, JHM 577

Schernhammer, ES 98

Scheulen, M 1480

Schinzari, G 1035

Schippinger, W 1021

Schleiermacher, G 238

Schmidt, C 1251

Schmidt, J 598

Schönthal, AH 1465

Schouten, JP 1457

Schouten, LJ 1291

Schultheis, B 1475

Schüz, J 986

Schwarz, SE 177

Scott, R 194

Scott, RJ 1225

Sebag-Montefiore, D 1333

Seid, PL 605

Selby, P 1063

Sellick, G 1305

Sellick, GS 1449

Senan, S 276

Serova, M 628

Seth, A 1690

Seth, R 133

Shack, LG 999

Shah, A 1009

Shamash, J 308, 1188

Shapiro, J 464

Sharp, L 133

Shastri, YM 1595

Shen, M-C 1577

Shenoy, H 1211

Shepherd, HL 6

Shi, X 218

Shiba, M 952

Shibata, A 426

Shibata, K 1538

Shibata, T 162

Shiiba, M 792

Shim, YM 22

Shimada, Y 170

Shin, HR 700

Shin, JY 605
Shin, SJ 458

Shinchi, H 405

Shipley, J 1707

Shirakusa, T 1648

Shirao, K 170

Shiratori, Y 1425

Shoda, H 1493

Shpall, EJ 391

Shun, K 941

Shuyama, K 85

Siapati, EK 210

Sidiropoulos, J 637

Siena, S 1469

Sier, CFM 398

Sierra, R 837

Siersema, PD 868

Sierzega, M 589

Sigal-Zafrani, B 1046

Sikes, RA 1713

Silvestrini, R 1499

Simpson, J 1310

Sinha, D 140

Skoglund, J 1175

Skubis-Zegadlło, J 531

Smallbone, K 646

Smith, A 1063, 1310

Smith, D 297

Smith, TAD 902

Smolarz, AJ 1552

Snijders, PJF 1457

Soares, FA 678

Sodek, KL 358

Soga, S 741

Sohaib, SA 1701

Soldan, K 989

Soliman, H 1157

Somanath, S 745

Sone, T 778

Song, B 1175

Song, CW 1523

Souckova, K 231

Souglakos, J 1560

Sousi, E 502

Spasojevic, I 735

Spector, TD 1696

Speizer, FE 1295

Sperduti, I 593, 1040

Spry, N 464

Squire, JA 678

Srivastava, G 1409

Srivastava, S 1690

Staibano, S 1545

Stampfer, MJ 98

Stanford, JL 826

Stanimirovic, A 1690

Stålberg, K 1583

Stathopoulos, E 1560

Stebbing, J 308, 1188

Steenbergen, RDM 1457

Steers, G 1277

Steevens, J 1291

Steger, GG 1021

Stein, JM 1595

Steinbild, S 1480

Steiner, J 844

Stevens, MCG 695

Steyerberg, EW 868

Stiggelbout, AM 717 
Stiller, CA 695

Stocken, DD 971

Stott, B 1381

Stovall, MA 695

Strand, A 129

Stratton, MR 1701

Strohfeldt, K 1234

Strumberg, D 1480

Stuart, N 384

Stupp, R 302

Suárez, C 201

Sugai, H 494

Sugar, L 1690

Sugimoto, H 1260

Suminoe, M 778

Summersgill, B 1707

Sun, BS 50

Sun, CK 50

Sun, J 1381

Sundstr $\varnothing \mathrm{m}$, SH 283

Sung, JJY 895

Sung, NY 700

Surowy, H 1184

Suttie, SA 902

Suzuki, H 426

Sweeney, NJ 1234

Syrigos, KN 670

Szczepanik, A 589

Szeszenia-Dạbrowska, N 832

Tabata, T 1058

Tacke, M 1234

Takahashi, N 1538

Takahashi, T 260

Takahashi, Y 851

Takaku, T 1099

Takao, S 405

Takayama, H 952

Takayama, T 851

Takeda, S 1260

Takeshima, N 1058

Takimoto, R 851

Takizawa, K 1058

Talamini, R 995

Talbot-Smith, A 686

Talekar, G 992

Tam, L 378

Tamakoshi, A 426

Tamatsukuri, S 1399

Tamesa, T 1399

Tamori, S 778

Tamura, T 162

Tanaka, F 543

Tanaka, YO 1053

Tanzawa, H 792

Tao, Q 895

Tao, Y 1664

Taoufik, E 1716

Taphoorn, MJB 302

Taraboletti, G 888

Tattersall, MHN 6

Taylor, I 502

Taylor, PR 123, 1567

Taylor, PRA 29

Te, V-C 1013

Tello, JM 1206

Terai, S 1399
Teramukai, S 37

Terauchi, T 1493

Terfloth, K 1329

Testa, D 1545

Tewfik, F 1625

Thaler, J 1021

Theodore, C 857

Therriault, H 1505

Thomas, C 238

Thomas, G 818

Thomas, H 844

Thomas, SB 1116

Thomsen, HF 1135

Thomson, CS 725, 1211

Thödtmann, R 1021

Thrasher, AJ 210

Thurieau, C 253

Thurston, DE 253

Thymara, I 1124

Tien, H-F 877

Timoshenko, AV 1090

Tirosh, B 1655

To, KF 895

Tobin, G 769

Tokuhisa, Y 1399

Toribio, RE 183

Torres, CH 678

Torrisi, R 802

Törnberg, S 129

Trachtenberg, J 1690

Tracy, E 1513

Traynor, P 378

Trehan, S 1625

Treluyer, J-M 290

Trepel, J 741

Tretiakova, MS 368

Trichopoulos, D 112, 1570

Trigila, N 1035

Tripaki, M 1560

Troise, F 1354

Troisi, R 688

Tsai, F-Y 334

Tschmelitsch, J 1021

Tsenga, A 1124

Tsou, T-C 334

Tsugane, S 446

Tsujimoto, G 260

Tsujimura, A 952

Tsunoda, H 1053

Tubiana-Mathieu, N 883

Tuman, RW 1344

Turley, H 1277

Turner, V 539

Turpin, E 1157

Tzehoval, E 1655

Uchida, K 1399

Ueno, H 145, 170

Ulivi, P 1499

Underhill, C 464

Uno, K 1053

Urayama, K 1315

Uzawa, K 792

Vaalburg, W 322

Vázquez, J 957

Vaccaro, A 1040

Vadai, E 1655
Valta, P 1028

Valteau-Couanet, D 238

Vamvakas, L 43

Van Calster, B 1194

Van Cutsem, E 1469

van Dam, P 659, 1165

van de Velde, CJH 717

van den Bent, MJ 302

van den Brandt, PA 1291

Van den Eynden, G 1165

Van den Eynden, GG 659

Van der Auwera, I 659, 1165

van der Deen, M 1077

van der Graaf, WTA 322

van der Hoeven, JJM 1084

van der Vijgh, WJF 1084

van der Zon, JM 398

van Duijn, W 398

van Dun, J 1344

van Emelen, K 1344

van Groeningen, CJ 1084

Van Hazel, G 464, 1469

Van Huffel, S 1194

Van Hummelen, P 1165

Van Laere, S 1165

Van Laere, SJ 659

Van Laethem, J-L 582

Van Marck, E 1165

Van Marck, EA 659

van Rietschoten, JGI 1457

van Vliet, EPM 868

Vandebroek, A 582

Varey, AHR 223

Varna, M 1157

Varshney, GC 345

Vassilopoulos, I 1124

Vatten, LJ 112

Vazquez, E 1683

Vecchia, CL 995, 1013

Velikova, G 1063

Venat-Bouvet, L 883

Ventouri, K 1124

Verborg, W 844

Vergote, I 1194

Verhage, BAJ 1291

Verhagen, CAHHVM 612

Verheij, M 712

Verheijen, JH 398

Vermeulen, PB 659, 1165

Veronesi, P 802

Verset, G 582

Verslype, C 582

Verspaget, HW 398

Verweij, J 1338

Vessey, M 434

Vetter, C 1271

Viale, G 802

Viloria, CG 201

Vincent-Salomon, A 1046

Virtanen, A 115

Vizoso, FJ 957

Vlahovic, G 735

Vogel, W 1184

Voigt, J-J 857

von Euler-Chelpin, M 1019

von Plessen, C 283

Voorzanger-Rousselot, N 964

Voutsina, A 1560
Vujaskovic, Z 735

Vyse, A 989

Wadell, G 129

Wagener, N 1271

Wakai, K 426

Wakelam, MJO 971

Wakeman, JA 384

Waller, J 691

Walpole, E 464

Wang, B-S 1577

Wang, H 420

Wang, J-C 941

Wang, S-f 420

Wang, W 745

Wang, X 1409, 1606

Wang, Y 1513

Wardle, J 691

Wartenberg, D 140

Watanabe, J 260

Watanabe, K 162

Waterfall, J 844

Watson, RW 1234

Waugh, N 133

Webb, EL 247

Wedrén, S 1287

Wegman, TD 322

Wei, L-H 1513

Weichert, W 523

Weiderpass, E 1570

Weiss Solís, D 818

Weitz, J 1146

Weller, C 523

Weller, D 1601

Weller, M 302

Wells, A 1361

Welsch, T 598

Welsh, BW 513

Werelius, B 1175

Whaley, D 1361

Willett, EV 1310

Williamson, P 670

Wilson, DGG 502

Wilson, P 308, 1188

Wilson, POG 670

Wilson, S 971

Wilting, SM 1457

Windebank, KP 29

Winter, DL 695

Winter, J 1486

Winther, JF 986

Witte, JS 557

Wohlmuth, P 1021

Wolf, M 1469

Wolk, A 429, 1005

Wolk, M 412

Wolpin, BM 98

Won, YJ 700

Wong, ML-Y 1409

Wong, N 1372

Wong, R 302

Wong, Y-C 1409

Wong, YP 895

Wood, K 29

Wood, W 1305

Woods, J 391

Woolf, K 472 
1724

Woolley, M 1469

Worrall, L 646

Wotherspoon, AC 949

Wright, P 1063

Wu, J 1441

Wyatt, P 308, 1188

Wynne, P 927

$\mathrm{Xu}, \mathrm{W} 741$

Yamada, A 1648

Yamada, Y 170

Yamaguchi, K 851

Yamamoto, E 1538

Yamanaka, T 37
Yamano, Y 792

Yamashita, Y 1648

Yanaga, K 543

Yang, L-Y 1690

Yang, W 1690

Yashiro, M 550

Yasui, H 170

Ye, H 949

Yeh, S-C 334

Yin, X 1315

Yokoe, H 792

Yokomine, T 405

Yokoyama, A 162

Yoshikawa, H 1053

Yoshimoto, M 678
Yoshimura, T 426

Yu, C-J 275

Yu, J 895

Yuen, H-F 1409

Zatonski, W 832

Zembala, M 589

Zentgraf, H 1271

Zgonjanin, L 735

Zhang, B-H 1577

Zhang, F 420

Zhang, L-y 420

Zhang, P 1664

Zhang, S 218

Zhang, XD 1225
Zhang, X-q 420

Zhang, Y 1099

Zhao, P 123

Zhi, J 1338

Zhou, X 1175

Zhou, Y 218

Zografos, G 73, 1716

Zoli, W 1499

Zou, L 1361

Zöller, M 1146

Zucchetti, M 888

Zucchetto, A 995

Zugmaier, G 1338

Zuidwijk, K 398

Zvereff, V 941 